\title{
Orbicularis oculi reflex in brain death
}

\author{
A. J. MEHTA AND S. S. SESHIA ${ }^{1}$ \\ From the Departments of Medicine and Paediatrics, University of Manitoba, Winnipeg, Manitoba, Canada
}

SYNOPSIS The early and late components of the orbicularis oculi reflex were absent bilaterally in three patients who fulfilled the clinical and electroencephalographic criteria of brain death. A response to peripheral facial nerve stimulation was obtained in all. These preliminary observations suggest that the study of the orbicularis oculi reflex is a simple, objective, bedside neurophysiological test of brain stem function, complementing the electroencephalogram in the diagnosis of brain death.

The criteria for the diagnosis of brain death, proposed by the Ad Hoc Committee of the Harvard Medical School (1968) have been widely accepted. The committee stressed the confirmatory value of the electroencephalogram showing electrocerebral silence. Such an electroencephalogram is not, by itself indicative of brain death, but is, in the absence of hypothermia and coma due to depressant drugs, strong evidence of cerebral or neocortical death (Report of the Ad Hoc Committee of the American Electroencephalographic Society on EEG Criteria for Determination of Cerebral Death, 1969; Brierley et al., 1971). Brain death implies irreversible loss of hemispheric and brain stem function and in view of the important social, medical, and legal implications of this diagnosis, a neurophysiological test of brain stem function would provide valuable corroborative evidence. Such a test is provided by the study of the orbicularis oculi reflex.

The evoked response in the orbicularis oculi muscle produced by electrical stimulation of the supraorbital nerve has two components, an early unilateral (R1) and a late bilateral (R2). The former has a latency of $10.6 \pm 2.5 \mathrm{~ms}$. The ipsilateral R2 component has a latency of $31 \pm 10 \mathrm{~ms}$, and the contralateral, $32 \pm 10 \mathrm{~ms}$ (Kimura, 1973). The afferent path of this, the orbicularis oculi reflex, is the trigeminal nerve and the efferent, the facial nerve. Kugelberg (1952) considered the

\footnotetext{
1 Address for reprint requests: Dr S. S. Seshia, Children's Centre, Winnipeg, Manitoba, Canada, R3E OW1.

(Accepted 16 April 1976.)
}

early response to be a myotatic reflex and the late, a polysynaptic one of cutaneous origin. Others (Tokunaga et al., 1958; Shahani and Young, 1968; Penders and Delwaide, 1973) have suggested a cutaneous origin for both components. The $\mathrm{R} 1$ response is abolished by destruction of $\frac{\mathrm{S}}{\mathrm{D}}$ 을 the principal sensory nucleus of the trigeminak nerve (Tokunaga et al., 1958) and may depend on an oligosynaptic pathway (Penders and Delwaide 1973). The $R 2$ response depends on a multi synaptic reflex arc which includes the spinal tracte of the trigeminal nerve (Kugelberg, 1952). While the central pathways of the reflex are not well understood, these must involve connections between the trigeminal and facial nuclei and the study of the orbicularis oculi reflex has been of value in evaluating brain stem integrity (Kimura, 1973).

This paper deals with our observations on the orbicularis oculi reflex in the diagnosis of brain death.

\section{METHODS}

The orbicularis oculi (blink) reflex was studied in three children who fulfilled the accepted criteria of brain death. These cases were admitted to the Intensive Care Unit of the Children's Centre, Winnipeg. They were assessed neurologically by one of us. Oculocephalic and oculovestibular reflexes, the latter using ice cold water, were tested in every case. The blink reflex study was carried out using a portable electromyograph machine (TECA Corporation-Model J). The supraorbital nerve was stimulated percutaneously with the cathode over the supraorbital foramen. 
The facial nerve was similarly stimulated with the cathode placed anterior to the mastoid process. Surface electrodes were applied over both orbicularis oculi muscles and reference electrodes on either side of the nose. The ground electrode was placed on the cheek. Where a response was obtained, supramaximal single shocks of $0.1 \mathrm{~ms}$ duration were used. When no responses were seen, the voltage was increased slowly to a maximum of $300 \mathrm{~V}$. Sweep durations of 30 and $100 \mathrm{~ms}$ and gains of $250 \mu \mathrm{V} / \mathrm{cm}$ were employed. The traces were displayed on an oscilloscope after conventional amplification. Electroencephalograms were recorded at gains of $7.5 \mathrm{~mm}=50 \mu \mathrm{V}, 1 \mathrm{~mm}=3 \mu \mathrm{V}$, and $1 \mathrm{~mm}=2 \mu \mathrm{V}$.

\section{CASE REPORTS}

CASE 1 (A.O.) This 11 year old boy was admitted to the Children's Centre on 6 November 1975, in cardiorespiratory arrest. This had followed a minor head trauma. After resuscitative measures, the heart beat and pulse returned. The duration of the arrest was estimated at 30 minutes. On examination, he was comatose, unresponsive, flaccid, and areflexic. Extraocular movements were absent and there was no spontaneous respiration. The blood pressure was labile and was maintained at normal levels with an isoprenaline infusion. The pupils, which were initially equal, $5 \mathrm{~mm}$, and non-reactive, dilated to $9 \mathrm{~mm}$ 11 hours after admission, but remained non-reactive. The findings otherwise remained unchanged until ventilatory support was withdrawn 48 hours after admission. Electroencephalograms two, 20, and 28 hours after admission showed electrocerebral silence. The blink reflex was studied 20 hours after admission. The early and late responses were absent on both sides. Direct stimulation of the facial nerve elicited an evoked response in the orbicularis oculi muscle (Table).

\section{TABLE}

LATENCY AND AMPLITUDE OF EVOKED MOTOR RESPONSE TO FACIAL NERVE STIMULATION

\begin{tabular}{cccccc}
\hline \multirow{2}{*}{ Case } & \multicolumn{2}{c}{ Right } & & \multicolumn{2}{c}{ Left } \\
\cline { 2 - 3 } \cline { 5 - 6 } & $(m s)$ & $(m V)$ & & $(m s)$ & $(m V)$ \\
\hline 1 & 4.1 & 2 & & 4.0 & 2 \\
2 & 4.0 & 2 & & 3.9 & 2 \\
3 & 3.6 & 1 & & 4.2 & 2 \\
\hline
\end{tabular}

Both components of the orbicularis oculi reflex were absent bilaterally in all three cases.
At necropsy, the brain was markedly swollen and soft in consisteney. There was significant bilateral uncal grooving. The right cerebellar hemisphere was soft and $5 \mathrm{~g}$ of dark red blood clot was found on its under surface. Microscopically, there was ischaemic necrosis of the major portion of the anterior pituitary. Prominent ischaemic or hypoxic change was evident in the motor cortex, globus pallidus, cortex of the insula, nuclei of the floor of the fourth ventricle, the dentate and olivary nuclei. A microscopic perivascular haemorrhage was seen in the floor of the fourth ventricle.

CASE 2 (T.C.) This 22 months old infant was admitted to the Children's Centre on 18 December 1975. She had been rescued from an apartment fire and found to be in cardiorespiratory arrest. Spontaneous respiratory effort and heart beat were restored after resuscitation. The duration of the arrest was estimated at 15 minutes. On admission, she was comatose and unresponsive. The tone was increased in all limbs, the deep reflexes were present, and the plantar responses flexor. She was triggering the respirator at rates of 35 to 40 per minute and ventilation was adequate. Shortly after admission, the pupils which were initially widely dilated and fixed, constricted to $5 \mathrm{~mm}$ but remained non-reactive. The blood carbon monoxide level was $22 \%$ on admission and $0 \%$ two hours later. Twenty-eight hours after admission, she continued to be comatose and unresponsive. Extraocular movements were absent. A few gasping respiratory efforts were observed. The pupils were $3 \mathrm{~mm}$ in diameter and reactive. The limbs were flaccid and areflexic except for the right upper which showed an increase of tone with preserved deep reflexes. The blink reflex study at this time showed absent early and late responses on both sides. Direct stimulation of the facial nerve resulted in an evoked response (Table). Forty-five hours after admission, she remained comatose and unresponsive. The extraocular movements were absent and the pupils were now widely dilated and fixed. The limbs were flaccid and areflexic. Some gasping respiratory efforts were present. An electroencephalogram showed electrocerebral silence. Seventy hours after admission, the findings remained unchanged and there was no spontaneous respiratory effort. An electroencephalogram was repeated and again showed electrocerebral silence. Respiratory support was withdrawn 77 hours after admission. A post-mortem examination was not obtained.

CASE 3 (S.A.) This 14 year old female was admitted to the Children's Centre on 8 December 1975. Twenty-four hours earlier, she had taken an overdose of acetylsalicylic acid. On admission, she was 
stuporous and confused but could be readily aroused. There were no abnormal neurological signs. The serum salicylate level was $1010 \mathrm{mg} / 1 \quad(101 \mathrm{mg} \%)$, blood $\mathrm{pH} 7.42, \mathrm{PO}_{2} 11.3 \mathrm{kPa}\left(\mathrm{PO}_{2} 86 \mathrm{mmHg}\right)$, $\mathrm{PCO}_{2} 2.7 \mathrm{kPa}\left(\mathrm{PCO}_{2} 20 \mathrm{mmHg}\right)$, and standard bicarbonate $15 \mathrm{mmol} / \mathrm{l}$. She was treated with peritoneal dialysis. On 10 December, the serum salicylate level was $320 \mathrm{mg} / \mathrm{l}(32 \mathrm{mg} \%)$ and blood gases and electrolytes were normal. She remained stuporous and confused. At 0130 on 11 December, she was given $5 \mathrm{mg}$ diazepam intramuscularly for restlessness. When seen 20 minutes later, she was well. At 0200 she was found in cardiorespiratory arrest. At this time, the pupils were widely dilated and fixed and no heart beat was audible. After resuscitative measures, normal cardiac rhythm was restored and the blood pressure stabilized at $100 / 80 \mathrm{mmHg}$. She was comatose, unresponsive, flaccid, areflexic, and had no spontaneous respiration. Ocular movements could not be elicited. The duration of the arrest was estimated at 10 minutes. The serum salicylate level was $130 \mathrm{mg} / \mathrm{l}(13 \mathrm{mg} \%)$. The findings remained unchanged for the next 34 hours and ventilatory support was withdrawn. Electroencephalograms eight and 32 hours after the episode of cardiorespiratory arrest showed electrocerebral silence. The orbicularis oculi reflex was studied 30 hours after the onset of coma. The early and late responses were absent on both sides but a response was obtained on direct stimulation of the facial nerve (Table).

At necropsy, the brain after fixation weighed $1340 \mathrm{~g}$. The cerebral convolutions were flattened and sulci narrowed. There was prominent bilateral uncal grooving. On microscopic examination, cerebral cortical neurones were well preserved. A few exhibited cytoplasmic eosinophilia. Neurones within the globus pallidus, midbrain, floor of the fourth ventricle, and cerebellum showed prominently increased eosinophilia of the cytoplasm and the nuclei were pale and smudged. Some neurones were represented as only eosinophilic 'ghosts'. In the cerebellum, the cells of the granular layer showed nuclear disintegration and there was exaggerated cytoplasmic eosinophilia of the Purkinje cells. Many of the cells of the dentate nucleus appeared as eosinophilic 'ghosts'. No microglial reaction was seen.

\section{DISCUSSION}

The early and late components of the orbicularis oculi reflex were absent bilaterally in all three patients studied. These children fulfilled the accepted criteria of brain death.
The $R 1$ response is affected by intrinsic and extrinsic lesions of the pons, whereas the R2 component is involved by lateral medullary lesions and its absence in coma may suggest diffuse suppression of the reticular system (Kimura, 1973). In a study on 48 comatose patients, Lyon et al. (1972) found the $\mathrm{R} 1$ response normal and the R2 absent in cases with supratentorial lesions. In two cases who developed transtentorial herniation, the $\mathrm{R} 1$ became unobtainable in one and was delayed bilaterally in the other. Cases 26 and 27 of their series are of interest. The $\mathrm{R} 1$ response was normal at a stage when they were obtunded. Following cardiorespiratory arrest, these patients became comatose with fixed dilated pupils, unelicitable brain stem reflexes, and inadequate spontaneous respiration. An electroencephalogram showed diffuse low voltage slow activity in one and the record was isoelectric in the other. Repeat blink reflex studies showed bilaterally absent early and late responses. Necropsy in one demonstrated severe homogenizing necrosis of neurones throughout the brain stem.

The significance of the absent orbicularis oculi reflex, like that of the electroencephalogram showing electrocerebral silence, depends upon the clinical situation. Both components of the reflex can be lost with combined hemispheric and brain stem dysfunction, and coma due to depressant drugs such as glutethimide and barbiturates may be associated with a reversible loss of both components of the reflex (Lyon et al., 1972). The reflex may be difficult to obtain during stages 2 to 4 of sleep (Kimura, 1973).

On the basis of our preliminary observations, we would suggest that, where the criteria of brain death exist, in the absence of hypothermia and coma due to depressant drugs or metabolic disturbance (if metabolic disturbances have been present, these should have been corrected at the time of the study) a bilateral absence of the early and late components of the orbicularis oculi reflex suggests that the brain stem is irreversibly involved.

We would like to thank Dr J. Hoogstraten, who performed the necropsies on cases 1 and 3, and Mrs S. Martin for secretarial assistance. 


\section{REFERENCES}

Brierley, J. B., Adams, J. H., Graham, D. I., and Simpson J. A. (1971). Neocortical death after cardiac arrest. Lancet, 2, 560-565.

Kimura, J. (1973). The blink reflex as a test for brain stem and higher central nervous system function. In New Developments in Electromyography and Clinical Neurophysiology, vol. 3, pp. 682-691. Edited by J. E. Desmedt. Karger: Basel.

Kugelberg, E. (1952). Facial reflexes. Brain, 75, 385-396,

Lyon, L. W., Kimura, J., and McCormick, W. F. (1972). Orbicularis oculi reflex in coma. Clinical, electrophysiological and pathological correlations. Journal of Neurology, Neurosurgery, and Psychiatry, 35, 582-588.

Penders, C. A., and Delwaide, P. J. (1973). Physiological approach to the human blink reflex. In New Developments in Electromyography and Clinical Neurophysiology, vol. 3, pp. 649-657. Edited by J. E. Desmedt. Karger: Basel.

Report of the Ad Hoc Committee of the Harvard Medical School to Examine the Definition of Brain Death (1968). A definition of irreversible coma. Journal of the American Medical Association, 205, 337-340.

Report of the Ad Hoc Committee of the American Electroencephalographic Society on EEG Criteria for Determination of Cerebral Death (1969). Cerebral death and the electroencephalogram. Journal of the American Medical Association, 209, 1505-1510.

Shahani, B., and Young, R. R. (1968). A note on blink reflexes. Journal of Physiology (London), 198, 103P-104P.

Tokunaga, A., Oka, M., Murao, T., Yokoi, H., Okumara, T., Hirata, T., Miyashita, Y., and Yoshitatsu, S. (1958). An experimental study on facial reflex by evoked electromyography. Medical Journal of Osaka University, 9, 397-411. 